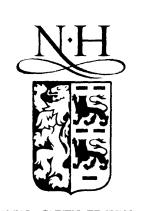

ELSEVIER

\title{
Model for sintering polydispersed glass particles
}

\author{
Miguel Oscar Prado ${ }^{\mathrm{a}, 1}$, Edgar Dutra Zanotto ${ }^{\mathrm{a}, *}$, Ralf Müller ${ }^{\mathrm{b}}$ \\ a Department of Materials Engineering - DEMa, Federal University of Sao Carlos-UFSCar, C.P. 676, CEP 13565-905, São Carlos, \\ SP, Brazil \\ ${ }^{\mathrm{b}}$ Federal Institute for Materials Research and Testing, Rudower Chausee 5, D-12489, Berlin, Germany
}

Received 22 June 2000

\begin{abstract}
We propose a model to describe the sintering kinetics of polydispersed glass particles, having no adjustable parameter. The model is based on three sintering stages: a pure 'Frenkel' (F) first step, a mixed 'Frenkel/MackenzieShuttleworth' stage, and a third, pure 'Mackenzie-Shuttleworth' (MS) step. The model considers sample shrinkage as the sum of the partial shrinkage of several clusters, each consisting of equally sized particles and each showing independent F or MS behavior. The overall set of clusters mimics the specimen's real particle size distribution. We then introduce the concept of neck forming ability $-\xi_{r}$, which allows the formation of necks among particles of different sizes, relaxing the clustering condition. Using experimental physical parameters: particle size distribution, viscosity, surface energy, and the theoretical $\xi_{r}$, the model describes well the sintering kinetics of an alumino-borosilicate glass powder having polydispersed, irregular shaped particles in a variety of temperatures. The sintering kinetics of the real powder is slower, but not far from the calculated kinetics of a monodispersed distribution containing only particles of average size. Thus the model provides a tool for estimating the sintering kinetics of real glass powders, for any size distribution and temperature, thus minimizing the number of laboratory experiments. (ㄷ 2001 Elsevier Science B.V. All rights reserved.
\end{abstract}

\section{Introduction}

The preparation of glass articles by sintering is a common practice in both the laboratory and the industry [1]. Models that predict the isothermal densification of a porous body, composed of glass particles having the same size or porous compacts having identical pores, as a function of viscosity,

\footnotetext{
* Corresponding author. Tel.: +55-16 2608250; fax: +55-16 2615404.

E-mail addresses: pradom@cab.cnea.gov.ar (M. Oscar Prado),dedz@power.ufscar.br (E. Dutra Zanotto).

${ }^{1}$ On leave from the Comisión Nacional de Energía Atómica, Centro Atómico Bariloche, 8400- SC de Bariloche, Argentina.
}

surface energy and particle size, successfully describe parts of the sintering process [2,3].

Several models have been proposed and many experiments conducted on sintering by viscous flow. Just to mention a few, numerical simulations, for instance, have also been applied to viscous sintering to study pore size distribution during solgel processing [4]. Exner et al. [5] developed a stereological model to describe the shrinkage on sintering glass powders. Their model will be discussed later in this paper. Giess et al. [2] reported that the pure MS analysis does not accurately describe the sintering of pressed compacts of polydispersed, irregular shaped glass particles, suggesting that this drawback may be the result of 
smaller particle-size fractions sintering more rapidly at the outset and larger ones sintering more slowly towards the end of the process.

We agree with Giess et al. in that no actual model describes the full sintering kinetics of real powdered glasses, having wide size distributions of particles. Thus, the objective of this paper is to present and test such a model, which takes into account the actual particle size distribution, and that considers a distribution of pore sizes and simultaneous Frenkel and MS processes at different points of the sample at any time.

\section{Summary of the theory}

The Frenkel model (Eqs. (1a) and (1b)) [6] describes well the isotropic sintering of monodispersed spherical particles. Starting from a loosely packed powder with a relative density of about 0.6 , the model works up to a relative density of $\rho=\rho(t) / \rho_{\mathrm{g}} 0.8$ (where $\rho(t)$ is the bulk density of the compact and $\rho_{\mathrm{g}}$ the glass density) or, equivalently, up to a linear shrinkage of approximately $10 \%$.

$\frac{\Delta L}{L_{0}}=\frac{3 \gamma}{8 \eta(T) r} t$

$\frac{\rho(t)}{\rho_{0}}=\left(1-\frac{3 \gamma t}{8 \eta(T) r}\right)^{-3}$

where $L_{0}$ represents the original sample length, $\Delta L$ the linear shrinkage after a sintering time $t, \eta(T)$ the temperature dependent shear viscosity, $\gamma$ the glass-vapor surface energy, $r$ the particle radius and $\rho_{0}$ is the relative green density.

For higher relative densities, when the pores are isolated, the Mackenzie-Shuttleworth, MS-model gives the densification rate [7]

$\frac{\mathrm{d} \rho}{\mathrm{d} t}=\frac{3 \gamma}{2 a_{0} \eta(T)}(1-\rho)$,

where $a_{0}$ is the initial radius of the pores, which is assumed to decrease during the final stage of sintering, while their number remains fixed.

On a laboratory time scale, sintering is only accomplished at temperatures above $T_{\mathrm{g}}$, where the viscosity curve $\eta(T)$ is normally well described by the Vogel-Fulcher-Tamman (VFT) equation [8]:

$\eta=\eta_{\infty} \mathrm{e}^{E_{\mathrm{v}} / R\left(T-T_{0}\right)}$,

where $R$ is the gas constant, $T_{0}$ an empirical constant, $E_{\mathrm{v}}$ the activation energy associated to molecular transport by viscous flow, and $\eta_{\infty}$ the viscosity at 'infinite' temperature. $T_{0}, E_{\mathrm{v}}$ and $\eta_{0}$ are obtained from shear viscosity measurements. Therefore, from Eqs. (1a), (1b), (2) for viscous flow sintering we expect that

$\left.\frac{\partial \rho}{\partial t}\right|_{\rho}=$ constant $\mathrm{e}^{-E_{\mathrm{V}} / R(T-T 0)}$.

Thus, the slope of a $\ln (\partial \rho / \partial t)$ vs. $\left(T-T_{0}\right)^{-1}$ plot gives the apparent activation energy $E_{\mathrm{v}} / R$.

\section{The clustering model}

Fig. 1 shows a SEM image of a compact of polydispersed particles after a linear shrinkage of about $8 \%$. Although the largest particles are clearly at the (initial) Frenkel stage, which is characterized by the formation of a neck between neighboring particles, the smaller particles (having a higher surface area and, thus, a higher driving force for sintering) have already overcome this stage. This is the basis for the model proposed here: small particles preferentially cluster in the open spaces left by larger particles and sinter faster. Thus, for a polydispersed compact with volume fraction $v_{r}$ of particles of radius $r$, we propose the following expression for the densification kinetics at a given temperature:

$$
\begin{aligned}
\rho(t)= & \sum_{r}\left[\rho_{\mathrm{F}}(r, t) \theta_{\mathrm{F}}\left(t_{0.8}-t\right)\right. \\
& \left.+\rho_{\mathrm{MS}}(r, t) \theta_{\mathrm{MS}}\left(t-t_{0.8}\right)\right] v_{r} \xi_{r} .
\end{aligned}
$$

Eq. (5) sums up the relative density $\rho(r, t)$ for each particle size, $r$, as a function of time, $t$. During the initial or Frenkel stage of sintering, the $\rho(r, t)<0.8$ condition is met and $\rho_{\mathrm{F}}(r, t)$ is calculated using the Frenkel equation (Eq. (1b)). Later, $\rho(r, t)>0.8, \rho_{\mathrm{MS}}(r, t)$ is calculated by the 

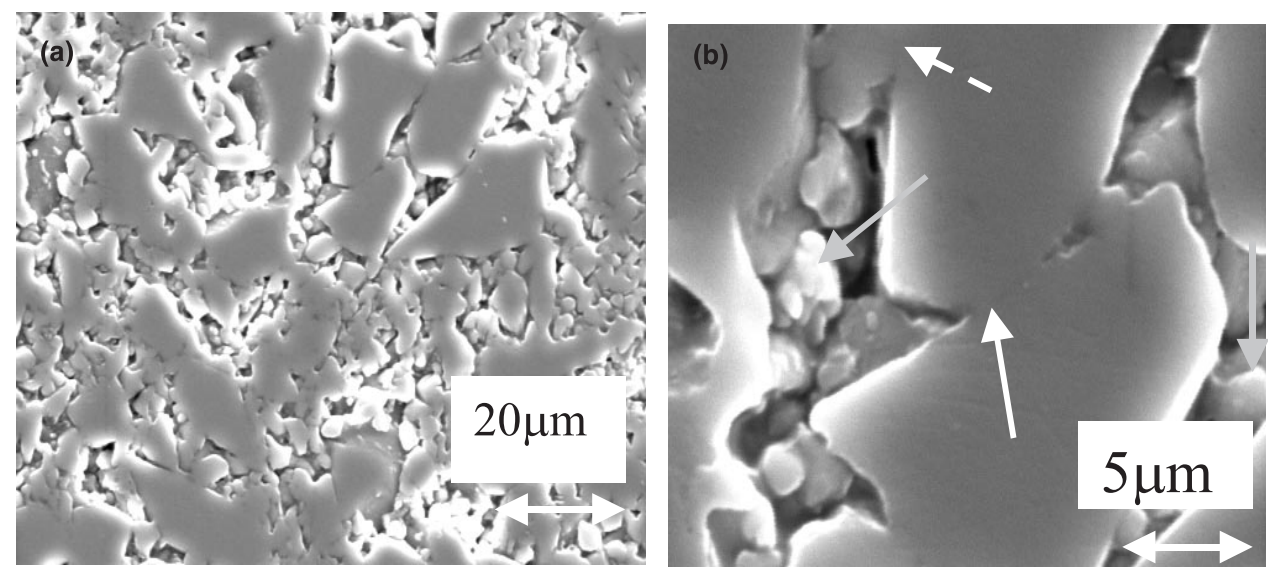

Fig. 1. SEM micrographs of a polydispersed compact after a linear shrinkage of $8 \%$. Magnification (a) $1200 \times$, (b) $5000 \times$ gray arrows: small sintered particles, white arrow: neck between two large particles, dashed white arrow: neck between a compact of small particles and a large particle.

Mackenzie-Shuttleworth model (Eq. (2)). For each cluster, the passage from the Frenkel regime to the MS regime is realized using the step functions $\theta_{\mathrm{F}}\left(t_{0.8}-t\right)$ and $\theta_{\mathrm{MS}}\left(t-t_{0.8}\right)$, whose values switch between 1 and 0 at $t=t_{0.8}$ when $\rho_{\mathrm{F}}\left(r, t_{0.8}\right)=0.8$ is reached. Thus, $\theta_{\mathrm{F}}\left(t_{0.8}-t\right)=1$ and $\theta_{\mathrm{MS}}\left(t-t_{0.8}\right)=0$ for $t<t_{0.8}$ and $\theta_{\mathrm{F}}\left(t_{0.8}-t\right)=0$ and $\theta_{\mathrm{MS}}\left(t-t_{0.8}\right)=1$ for $t>t_{0.8}$.

The pore radius $a_{0}$ in Eq. (2) was adjusted to guarantee a continuous $\rho(r, t)$ function at $t=t_{0.8}$. The adjustment was achieved by first computing $t_{0.8}$ with Eq. (1b); then calculating $a_{0}$ with the integrated version of Eq. (2), at $t=t_{0.8}$.

The (theoretical) function $\xi_{r}$ considers the formation of necks among particles of different radii (Appendix A). For each particle size $r, \xi_{r}$ is a normalized overall average of the number of necks these particles can form with any other particle size of the considered distribution of sizes, and can be calculated as

$\xi_{r}=\frac{1 / r^{\mathrm{c}}}{\sum_{r} v_{r} / r^{\mathrm{c}}}$

where $r$ is the particle radius, $v_{r}$ the volume fraction of particles with radius $r$, and the exponent $\mathbf{c}$ is calculated from the particle size distribution using Eq. (7). $\ln \left(\sum_{i} n\left(r, r_{i}\right) v_{r_{i}}\right)=\mathrm{constant}-c \ln (r)$,

where $n\left(r, r_{i}\right)$ is the number of particles of radius $r$ that can be accommodated around a particle of radius $r_{i}$. The theoretical basis of Eq. (7) is given in Appendix A.

For the size distribution shown in Fig. 2, the calculated value of $\mathbf{c}$ is $\mathbf{1 . 2 3}$. Thus, by introducing $\xi$, the model does not rely on any adjustable parameter. Eq. (5) can be explicitly written as Eq. (8)

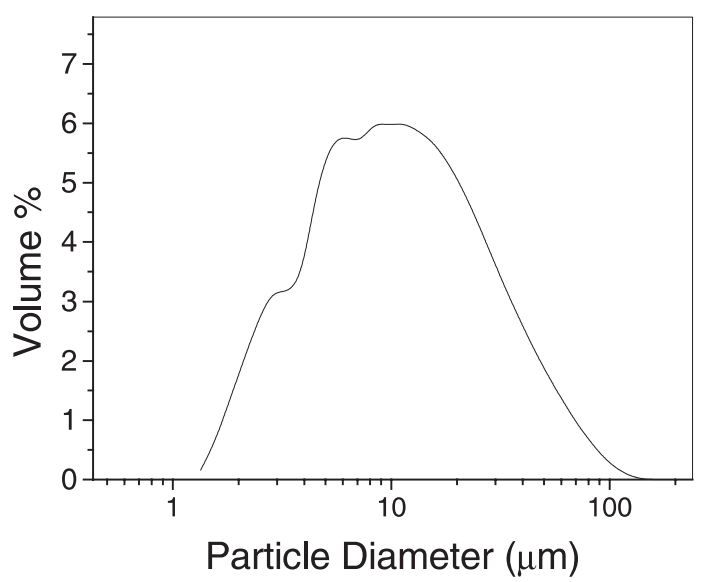

Fig. 2. Particle size distribution of the glass powder used in this work, measured with a Mastersizer $\mu$ Ver.2.0. 


$$
\begin{aligned}
\rho(t)= & \sum_{r}\left(\frac{\rho_{0}}{\rho_{\mathrm{g}}\left(1-\frac{3 \gamma t}{8 \eta(T) r}\right)^{3}} \theta\left(t_{0.8}-t\right)\right. \\
& +\left(1-\exp \left(\frac{-3 \gamma t}{2 a_{0}(r) \eta(T)}+\ln \left(1-\frac{\rho_{0}}{\rho_{\mathrm{g}}}\right)\right)\right) \\
& \left.\times \theta\left(t-t_{0.8}\right)\right) v_{r} \xi_{r} .
\end{aligned}
$$

To test the proposed model, we used a polydispersed, irregular shaped glass powder and determined the densities of uniaxially die-pressed cylindrical samples by weighing and measuring their diameters and heights after sequential isothermal heat treatments. The relative densification rate $(\partial \rho / \partial t)$ was obtained from both linear shrinkage and buoyancy measurements as a function of time for various sintering temperatures. A corrected sintering time was introduced to allow for the retarded thermal equilibrium in the sample bulk. For all stages of sintering, $\ln (\partial \rho / \partial t)$ showed a linear dependence on $\left(T-T_{0}\right)^{-1}$, where $T$ is the actual sintering temperature and $T_{0}$ an empirical constant, indicating a process controlled by viscous flow, as expected. A marked sintering anisotropy was found, ascribed to particle orientation during pressing. The sintering curves at different temperatures follow a unique 'master curve' when plotted against the reduced time $\gamma$. $t / \eta$, where $t$ is the sintering time, $\eta$ the shear viscosity and $\gamma$ the glass-vapor surface energy. The sintering of polydispersed powders is not satisfactorily described by the existing models for monodispersed particles. Necks form between different sized particles and particle clustering occurs. The clustering model provides a good description of the sintering kinetics of this powder. Our experiments and results are described in more detail in the following sections.

\section{Materials and methods}

An alumino-borosilicate glass of composition (wt\%): $71.70 \mathrm{SiO}_{2}, 8.33 \mathrm{~B}_{2} \mathrm{O}_{3}, 8.56 \mathrm{Al}_{2} \mathrm{O}_{3}, 1.00$
$\mathrm{MgO}, 2.67 \mathrm{CaO}, 7.44 \mathrm{Na}_{2} \mathrm{O}$, which is a candidate for nuclear waste encapsulation, was used owing to its stability against devitrification and resistance to water corrosion $[9,10]$. Due to its high liquidus temperature $(1820 \mathrm{~K})$, the final compact, including nuclear wastes, should be prepared by sintering at lower temperatures to avoid hazardous volatiles leaving the glass matrix and contaminating the surrounding atmosphere. Therefore, this glass was used not only to test the clustering model but also because its sintering behavior is technologically significant.

The glass transition temperature, $T_{\mathrm{g}} \approx 845 \mathrm{~K}$, was determined by differential scanning calorimetry at $10 \mathrm{~K} / \mathrm{min}$. Fig. 2 shows the particle size distribution measured with a Mastersizer $\mu$ Ver.2.0.

High temperature viscosity measurements were taken using a rotating cylinder viscometer. Assuming that $\eta_{T_{\mathrm{g}}} \approx 10^{12.5} \mathrm{~Pa} \mathrm{~s}$ at the glass transition temperature $T_{\mathrm{g}}^{\mathrm{DSC}}$, the viscosity showed a VogelFulcher-Tamman behavior with $\eta_{\infty}=\mathrm{e}^{-3.9681} \mathrm{~Pa} \mathrm{~s}$, $E_{\mathrm{v}}=83.7 \mathrm{~kJ} / \mathrm{mol}$ and $T_{0}=562.48 \mathrm{~K}$.

The glass-vapor surface energy $\gamma$ varied slightly with temperature, but there is no available data for our glass for temperatures near $T_{\mathrm{g}}$, at which the sintering experiments were performed. Hence, extrapolated data was used employing Lyon's method [11]. The values of $\gamma$ extend from 0.327 $\mathrm{J} / \mathrm{m}^{2}$ at $959 \mathrm{~K}$ to $0.325 \mathrm{~J} / \mathrm{m}^{2}$ at $1017 \mathrm{~K}$.

Cylindrical powder compacts were prepared by uniaxial die-pressing of around $0.75 \mathrm{~g}$ of sample glass powder at $0.5 \mathrm{MPa}$ for $30 \mathrm{~s}$. The cylinders were approximately $6 \mathrm{~mm}$ height and $10.2 \mathrm{~mm}$ in diameter. The sample's length, $l$, and diameter, $\phi$, were measured after each sequential isothermal sintering step to determine its relative density. When the density did not further increase with heat treatment, final compact density was also determined by the Archimedes method, using liquid mercury.

Horizontal dilatometry was attempted to follow the sintering kinetics [12]; however, this technique was discarded due to the significant creep caused by the pressure of the measuring bar on the sample. The fact that the samples did not reach thermal equilibrium instantaneously when brought to the sintering temperature was considered. The corrections performed are detailed in Appendix B. 
The sequence of experiments and calculations were:

(a) Measurement of densification as a function of corrected time, $t^{*}$, at each temperature: 959 , 966, 980, 989, 999 and $1017 \mathrm{~K}$ (see Appendix B for the meaning of $t^{*}$ ).

(b) Calculation of the densification rate $\partial \rho / \partial t^{*}$ vs. time for each temperature.

(c) Construction of $\ln \left(\partial \rho / \partial t^{*}\right)$ vs. $\rho$ curves.

(d) Determination of $\ln \left(\partial \rho / \partial t^{*}\right)$ vs. $\left(T-T_{0}\right)^{-1}$ at constant $\rho$.

(e) Determination of the activation energies $E_{\mathrm{v}}$ from the curve slopes at (d).

(f) Calculation of mass density as a function of time, $t^{*}$, at each temperature: 959, 966, 980, 989, 999, $1017 \mathrm{~K}$ (densification curves) using the present model.

\section{Results}

Fig. 3 shows the sintering curves at different temperatures plotted against the reduced time, $\gamma t^{*} / \eta$. The reasonable match among these curves confirms that glass sintering depends on temperature mainly through viscosity.

Fig. 4 shows the axial and radial fractional shrinkage for all temperatures as a function of the reduced time. A marked anisotropy is evident, with radial shrinkage about $25 \%$ higher than axial

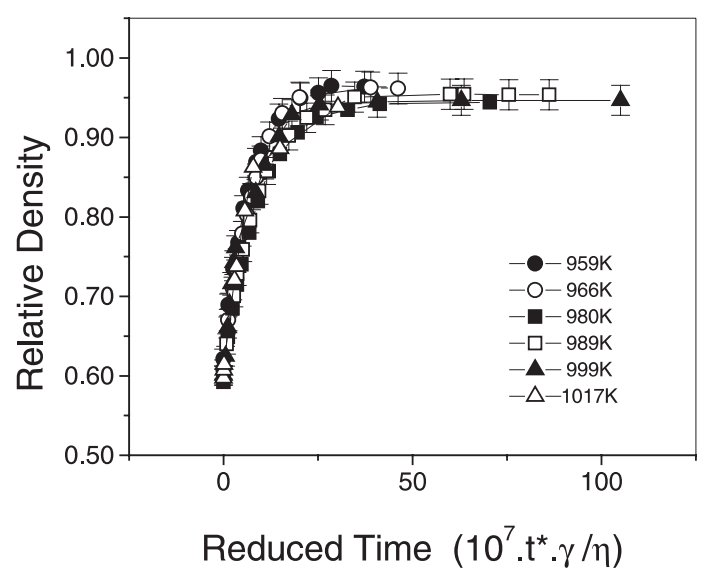

Fig. 3. Sintering curves at 959, 966, 980, 989, 999 and $1017 \mathrm{~K}$ as a function of the reduced time $10^{7} \gamma t^{*} / \eta$.

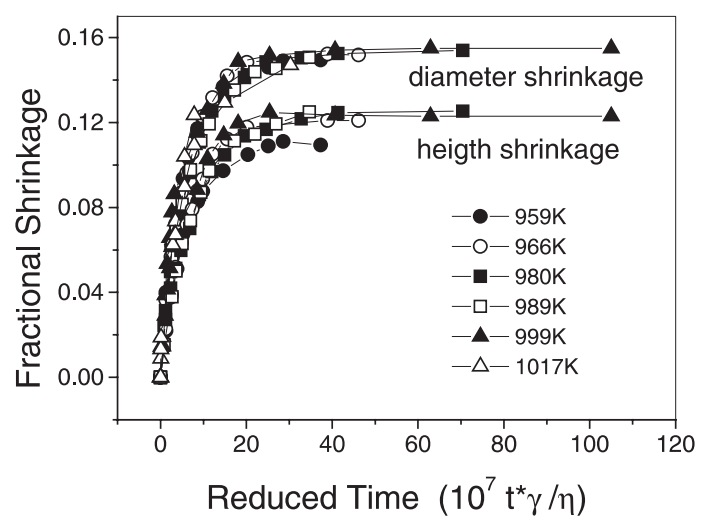

Fig. 4. Fractional height and diameter shrinkage for all temperatures used in this study.

shrinkage. To gain a better understanding of the real sintering process, we have included the sintering curves of five idealized monodispersed powders in Fig. 5. A comparison of the kinetics of these idealized processes with the real one highlights the effect of having a polydispersed distribution. This figure shows the calculated sintering curves for a monodispersed distribution of the smallest particles (curve a), a monodispersed distribution of the average particle size (curve b) and a monodispersed distribution of the largest particles, according to the measured particle size distribution shown in Fig. 2. The curves were calculated with the clustering model with $\mathbf{c}=0$

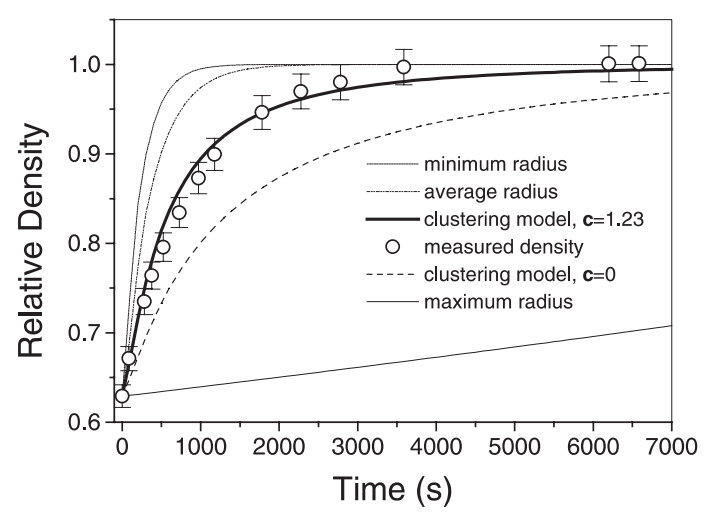

Fig. 5. Relative density vs. time at $989 \mathrm{~K}$, showing the sintering kinetics of: (a) smallest particles; (b) average particles; (c) clustering model with $\mathbf{c}=1.23$; (d) clustering model with $\mathbf{c}=0$; (e) largest particles. 


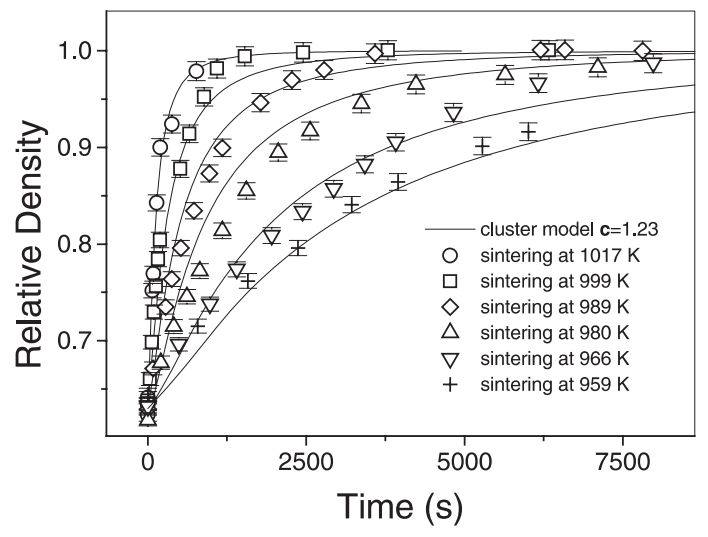

Fig. 6. Relative density, $\rho\left(t^{*}\right)$, for different sintering temperatures. The symbols represent measured values. The continuous lines are calculated from the clustering model.

(curve $\mathrm{d}$ ) and the calculated value of $\mathbf{c}=1.23$ (curve c). The sintering kinetics of the real distribution is closer to that of the average size particles.

Since our samples never reached $\rho=1$, but only reached $\rho_{\mathrm{f}}=0.96$ at the final stage of sintering (due to the pressure of the entrapped gases in the closed pores, which reach an equilibrium diameter at each temperature), the final density $\rho_{\mathrm{f}} \approx 0.96 \rho_{\mathrm{g}}$ was used throughout our calculations instead of $\rho_{\mathrm{g} .}$

Fig. 5 shows the measured shrinkage at $989 \mathrm{~K}$ of the polydispersed powder whose size distribution curve is shown Fig. 2. Fig. 6 shows other sintering curves from which the activation energy for viscous flow $E_{\mathrm{V}}$ was calculated. The results are given in Fig. 7. The average value is $E_{\mathrm{v}}=81.3 \mathrm{~kJ} / \mathrm{mol}$.

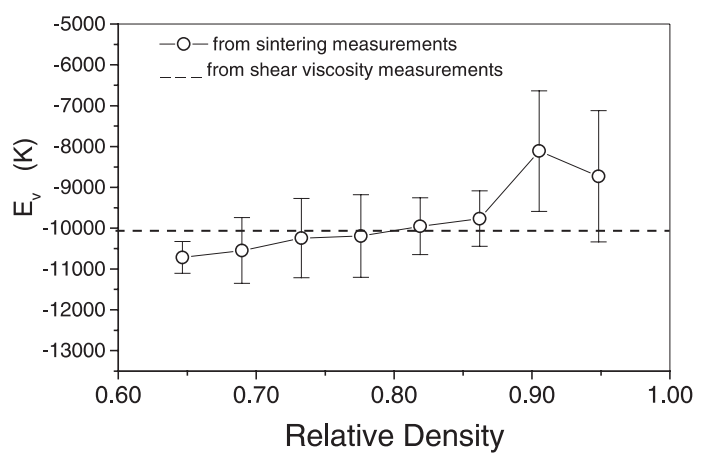

Fig. 7. Activation energy vs. relative density from sintering experiments and from shear viscosity measurements.

\section{Discussion}

The superimposition of all the sintering curves (Fig. 3) within experimental error when plotted against the corrected reduced time $\gamma t^{*} / \eta$ (see Appendix $\mathrm{B}$ for $t^{*}$ ) confirms that sintering is controlled by viscous flow and by the absence of surface crystallization of the powder. It also provides a test for the accuracy of our time, temperature and density measurements.

We assign the anisotropy shown in Fig. 4 to a geometrical rearrangement of the particles during uniaxial pressing. The flat sides of the (irregular) particles tend to align horizontally, leaving the acute ends of high curvature pointing radially. This is equivalent to the treatment of Giess et al. [2].

The cluster model proposed here Eq. (5) is a natural way of expressing the sintering of a polydispersed distribution of particles with the simplest existing models (F \& MS) as a basis. As small particle clusters sinter faster than larger particle clusters, the model allows for regions of Frenkel behavior and MS behavior to occur simultaneously. It not only considers the simultaneous sintering of clusters of particles with a distribution of sizes but also includes a distribution of pore sizes and the formation of necks between particles with different radii through the $\xi_{r}$ function without any adjustable factor.

The $\mathbf{c}$ parameter depends on the shape and size distribution of the particles. If $\mathbf{c}=0$, there is no mixing of particles of different sizes. When $\mathbf{c}>0$, particles of different sizes are allowed to form necks, as illustrated in Fig. 1, which becomes evident by the results shown in Fig. 5. For glasses in which crystallization does not occur (crystallization can hinder viscous flow sintering $[13,14]$ ), predictions can be made with the model for other temperatures. This fact has practical importance, since previously anticipates sintering results from simple calculations.

The sintering kinetics of the real powder is slower, but not far from the calculated kinetics of a monodispersed distribution containing only particles of average size. The model provides a tool for estimating the sintering kinetics of real glass powders, for any size distribution and tempera- 
ture, thus minimizing the number of laboratory experiments.

When the sample size is sufficiently large to create significant temperature gradients, these gradients must be taken into account. We propose Eq. (B.1) as a simple and effective way to perform such a correction. Proof that this is an adequate procedure is the agreement between the activation energy $E_{\mathrm{v}}=81 \pm 3 \mathrm{~kJ} / \mathrm{mol}$ from sintering measurements against $E_{\mathrm{v}}=84 \pm 1 \mathrm{~kJ} / \mathrm{mol}$ obtained independently by fitting the VFT relationship from shear viscosity measurements.

Exner's model [5], derived using stereological equations to describe the pore/solid interface during sintering, has the form

$\ln \left(1-\frac{\Delta L}{\Delta L_{\mathrm{f}}}\right)=\frac{-2 \pi \gamma}{3 \eta(T) \lambda_{0}} \frac{1}{\Delta L_{\mathrm{f}} / L_{0}}\left(t-t_{0}\right)$

$\lambda_{0}=4 \frac{V_{\mathrm{S}}}{S_{0}}$,

where $\Delta L_{\mathrm{f}}$ is the total shrinkage after sintering to full density, $L_{0}$ the length of the compact before sintering, and $\lambda_{0}$ is related to the surface $\left(S_{0}\right) /$ volume $\left(V_{\mathrm{S}}\right)$ ratio by the equation.

The calculated $\ln \left(1-\Delta L / \Delta L_{\mathrm{f}}\right)$ curves (using the present model) are shown in Fig. 8 for monodispersed and polydispersed distributions. The behavior of a monodispersed system was calculated for a particle size equal to the average of the po-

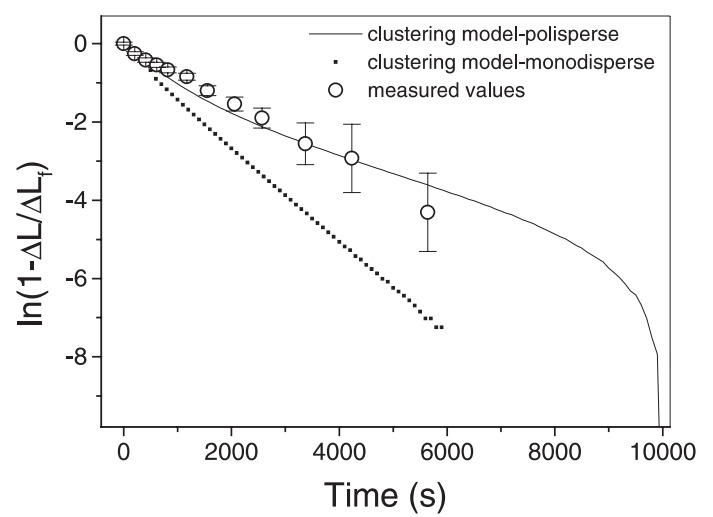

Fig. $8 . \ln \left(1-\Delta L / \Delta L_{\mathrm{f}}\right)$ vs. time calculated for: the glass with polydispersed size distribution; for a monodispersed distribution with particle size corresponding to the average of the polydispersed one and measured values. All data at $980 \mathrm{~K}$. lydispersed distribution. Fig. 8 shows departures from Exner's model, however, the scatter of the measurements did not allow us to prove this prediction.

\section{Conclusions}

A new model was proposed to describe the sintering kinetics of polydispersed irregular glass particles. The model assumes the clustering of equally sized particles, the formation of necks among particles of different sizes, a distribution of pore sizes and the simultaneous occurrence of Frenkel and Mackenzie-Shuttleworth sintering. While the sintering kinetics is not well described by either the $\mathrm{F}$ or the MS equations for average sized particles, the new model provides a good description of the full sintering kinetics using no adjustable parameter for all temperatures. This goal was achieved through the introduction of the function $\xi_{r}$ which is readily calculated for any particle size distribution.

To test the model, we studied the sintering of a polydispersed alumino-borosilicate glass powder of irregular particles. The experimental isothermal $\rho(t)$ vs. $\gamma t^{*} / \eta$ curve followed a unique pattern at all temperatures. The activation energy, $E_{\mathrm{v}}=81 \pm 3$ $\mathrm{kJ} / \mathrm{mol}$, agrees with $E_{\mathrm{v}}=84 \pm 1 \mathrm{~kJ} / \mathrm{mol}$ obtained by fitting the VFT equation from shear viscosity measurements.

The sintering kinetics of the real powder is slower, but not far from the calculated kinetics of a monodispersed distribution containing only particles of average size. Thus the model provides a tool for estimating the sintering kinetics of real glass powders, for any size distribution and temperature, thus minimizing the number of laboratory experiments.

\section{Acknowledgements}

This work was funded by grants from the Consejo Nacional de Investigaciones Científicas y Técnicas (Argentina), CNPq, Pronex and FAPESP (Brazil). 


\section{Appendix A. Geometrical considerations about neck formation among particles of different radii}

As shown by Frenkel Eqs. (1a) and (1b), the sintering velocity depends on physical parameters $(\gamma, \eta)$ and on particle size. In the case of a polydispersed distribution, necks form among particles of different sizes, as shown in Fig. 1. The problem of counting exactly how many contacts are formed among particles of different radii is very complex and, to our knowledge, there is no theoretical expression for the sintering kinetics of (irregular) particles of different sizes. We do know, however, that a mixture of a certain volume of particles of radii $r_{1}$ with the same volume of particles of radii $r_{2}$ will sinter at a velocity that is not the average of the velocity corresponding to $r_{1}$ or $r_{2}$, but is closer to that of the smaller particles (Fig. 5). This is reasonable considering the fact that the smaller particles have a larger surface energy and, thus, a larger driving force for sintering.

We now introduce a weighing function, $\xi_{r}$ (as used in Eq. (7)), whose value depends on the particle size and that weighs the particle volume fractions $v_{r}\left(v_{r}^{\prime}=v_{r} \xi_{r}\right)$. This $\xi_{r}$ should be larger for the smallest particles since the actual sintering rate is closer to that of the smaller particles. We propose that the variation of $\xi_{r}$ with the particle radius $r$, is the same as the variation of the number of necks that particles of radius $r$ can form around a particle of radius $r_{0}$ averaged over all $r_{0}$ values for the distribution studied.

The next question, then, is 'how many particles of radius $r$ can be accommodated around a particle of radius $r_{0}$ ?' For the sake of simplicity, we will consider a compact array of spherical particles of radius $r$ at the surface of a spherical particle of radius $r_{0}$ (shown in Fig. 9).

From simple geometrical considerations, the angle $\alpha$ stretched by a particle of radius $r$ in contact with a particle of radius $r_{0}$ (Fig. 9) is given by Eq. (A.1).

$$
\alpha=2 a \tan \frac{1}{\sqrt{\left(\frac{r_{0}}{r}\right)^{2}+2 \frac{r_{0}}{r}}} .
$$

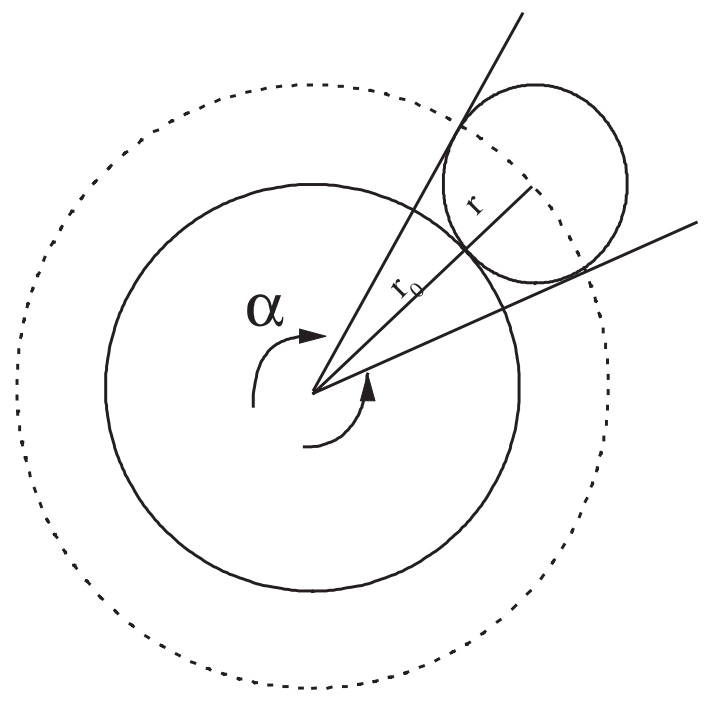

Fig. 9. Particle of radius $r$ forming a neck with a particle of radius $r_{0}$. The dotted line shows an imaginary spherical surface with radius $r+r_{0}$.

So the number $n\left(r, r_{0}\right)$ of particles of radius $r$ that can be accommodated around a particle of radius $r_{0}$ is

$n\left(r, r_{0}\right) \approx \frac{4 \pi\left(r_{0}+r\right)^{2}}{\left(\alpha\left(r_{0}+r\right)\right)^{2}} \frac{2}{\sqrt{3}}=\frac{8 \pi}{\sqrt{3}} \frac{1}{\alpha^{2}}$,

where $4 \pi\left(r_{0}+r\right)^{2}$ is the area of a sphere with radius $\left(r_{0}+r\right), \alpha^{2}\left(r_{0}+r\right)^{2}$ is the area occupied by each surrounding particle, and the factor $2 / 3^{1 / 2}$ accounts for a closed packed arrangement of surrounding particles at the surface of the central particle.

The arc-tangent function in Eq. (A.2) can be approximated by Eq. (A.3)

$\frac{1}{(a \tan (x))^{2}} \approx \frac{2}{3}+2 x^{-2}-\frac{1}{15} x^{2}$,

where $0<x<1$. This condition implies $0<r<$ $2.5 r_{0}$ in Eq. (A.1).

The argument $x$ can be written as a function of $r$ and $r_{0}$, inserting Eq. (A.1) into Eq. (A.3). If one remains restricted to $r<r_{0}$, then one can develop Eq. (A.3) in series. 
$\frac{1}{(a \tan (x))^{2}} \approx \frac{2}{3}+2 \frac{r_{0}}{r}+\left(\frac{r_{0}}{r}\right)^{2}$.

Keeping only the first three terms, one has:

Using Eqs. (A.2) and (A.4) one obtains Eq. (A.5)

$n\left(r, r_{0}\right) \approx \frac{2 \pi}{\sqrt{3}}\left(\frac{2}{3}+2 \frac{r_{0}}{r}+\left(\frac{r_{0}}{r}\right)^{2}\right)$.

From Eq. (A.5), one can see that $n(r)$ depends on $r$ as a mixture of negative powers -2 and -1 . In practice, one observes that the dependence of $\boldsymbol{n}$ on $\mathbf{r}$ for a fixed value of $r_{0}$ can be roughly expressed as $1 / r^{\mathbf{c}}(1<\mathbf{c}<2)$. Hence, the number of particles that surround a central particle increases with decreasing $r$ as $1 / r^{\mathbf{c}}$.

A weighing function $\xi_{r}$ is now introduced corresponding to particles with radius $r$

$\xi_{r}=\frac{1 / r^{c}}{\sum_{r} v_{r} / r^{c}}$

within a particle size distribution. For a given size distribution and particle geometry, $\mathbf{c}$ is a theoretical constant, and its value is determined by equating the overall volume fraction

$\ln \left(\sum_{i} n\left(r, r_{i}\right) v_{r_{i}}\right)=\mathrm{constant}-c \ln (r)$

weighed average of the $n\left(r, r_{0}\right)$ functions for different $r_{0}$ values to a $1 / r^{c}$ behavior, as shown in Eq. (A.7).

From Eq. (A.6) it is possible to demonstrate that the following relations are met:

$$
\begin{aligned}
& \sum_{r} v_{r} \xi_{r}=1, \\
& \sum_{r} v_{r}=1 .
\end{aligned}
$$

\section{Appendix B. Sample temperature measurements}

When a cold glass sample is introduced into a furnace previously heated to a $T_{\text {sint }}$ temperature, each of its volume elements requires a different

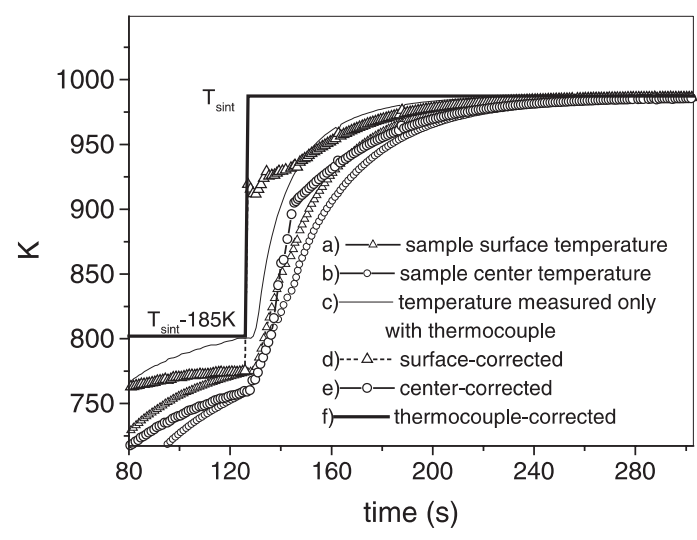

Fig. 10. Temperatures measured at: (a) the sample surface, (b) the sample center (c) thermocouple and sample holder. (d)-(f) same as (a)-(c) with correction for thermocouple delay time.

length of time to reach that temperature. The center of the sample obviously needs the longest time.

Fig. 10 shows the measured temperature at the sample's surface and center during sintering. Therefore, in assigning the actual sintering times $t^{*}$, the measured times, $t$, were weighed by the factor

$t^{*}=t \frac{\mathrm{e}^{E_{\mathrm{v}} /\left(T_{\mathrm{s}} \text { int }-T_{0}\right)}}{\mathrm{e}^{E_{\mathrm{v}} /\left(T_{\mathrm{s}} \text { int }-\Delta T-T_{0}\right)}}$,

where $T_{\text {sint }}-\Delta T$ is the actual temperature of the sample at time $t$ while reaching thermal equilibrium, $(\Delta T>0)$ and $T_{0}$ is the VFT temperature.

This manner of weighing the sintering time intervals comes straight from Eqs. (1a) and (1b), in which $t$ is proportional to $\eta$. In this study, we made this correction considering the evolution of center and surface temperatures of the sample (both measured, Fig. 3) and assuming, for a first approximation, a linear temperature distribution from the center to the surface of the glass cylinders.

\section{References}

[1] E.M. Rabinovich, J. Mat. Sci. 20 (1985) 4259.

[2] E.A. Giess, J.P. Fletcher, L. Wynn Herron, J. Am. Ceram. Soc. 67 (8) (1984) 549. 
[3] a)Yet-Ming Chiang, D.P. Birnie III, W.D. Kingery, in: Physical Ceramics. Principles for Ceramic Science and Engineering, Wiley, New York, 1997, p. 392.

[4] A. Van de Vorst, J. Am. Ceram. Soc. 81 (8) (1998) 2147.

[5] H.E. Exner, E.A. Giess, A Stereology-Based Equation for Isotropic Shrinkage during Sintering by Viscous Flow, Science of Sintering Seventh, Plenum, New York, 1989, p. 73 .

[6] J. Frenkel, J. Phys. (USSR) IX (5) (1945) 385.

[7] J.K. Mackenzie, R. Shuttleworth, Proc. Phys. Soc (London) 62 (1949) $833 \mathrm{Sec}$. B.

[8] I. Gutzow, J. Schmelzer, in: The Vitreous State. Thermodynamics, Structure, Rheology and Crystallization, Springer, Berlin, 1995, p. 32.

[9] M.A. Audero, A.M. Bevilacqua, N.B. Messi de Bernasconi, D.O. Russo, M.E. Sterba, J. Nucl. Mat. 223 (1995) 151.
[10] A.M. Bevilacqua, N.B. Messi de Bernasconi, D.O. Russo, M.A. Audero, M.E. Sterba, A.D. Heredia, J. Nucl. Mat. 229 (1996) 187.

[11] O.V. Mazurin, M.V. Streltsina, T.P. Shvaiko-Shvaikovskaya, V.K. Leko, A.I. Priven, SciGlassTM 3.0, Universal Information System on Glass Properties. scivision@delphi.com http://www.scivision.com.

[12] A.R. Bocaccini, Journal of Materials Research 13 (6) (1998) 1693.

[13] R. Müller, in: Glastech. Ber. Glass Sci. Technol.: Proceedings of Fifth International Otto Schott Coll., Jena FRG, 67C, 1994, p. 93.

[14] I. Gutzow, R. Pascova, A. Karamanov, J. Schmelzer, in: Glastech. Ber. Glass Sci. Technol. Proceedings of Fifth International Otto Schott Coll., Jena FRG, 67C, 1994, p. 81. 